TRANSACTIONS OF THE

AMERICAN MATHEMATICAL SOCIETY

Volume 172, October 1972

\title{
ON LIE'S THEOREM IN OPERATOR ALGEBRAS
}

\author{
BY
}

\section{F.-H. VASILESCU}

\begin{abstract}
This work contains some algebraic results concerning infinite dimensional Lie algebras, as well as further statements within a topological background. Natural generalizations of the notion of radical, solvable and semisimple Lie algebra are introduced. The last part deals with variants of a Lie's theorem in operator algebras.
\end{abstract}

1. Introduction. Let $L$ be a Lie algebra, not necessarily finite dimensional, over a field $K$. Denote by $\left\{L^{(n)}\right\}, n \geq 0$, the derived series, i.e. $L^{(0)}=L, L^{(n+1)}$ $=\left[L^{(n)}, L^{(n)}\right], n>0$. We recall that $L$ is said to be solvable when $L^{(n)}=0$ for a certain $n$. It is well known [3] that every subalgebra and every homomorphic image of a solvable Lie algebra is solvable. Also the sum of two solvable ideals is a solvable ideal. In particular, when $L$ is finite dimensional, there is a solvable ideal $R_{L}$ of $L$ which contains any other solvable ideal of $L$ and this is the radical of $L$. In what follows we attempt to construct a radical for a not necessarily finite dimensional Lie algebra as an ideal $R_{L}$ of $L$ which contains any solvable ideal of $L$ such that the quotient $L / R_{L}$ has 0 as radical. Moreover, $R_{L}$ is the smallest ideal in $L$ with the described properties. We mention that there are some other constructions of a radical [6] but our point of view seems quite different. As we have a radical, we may define the notion of semisimple Lie algebra. It turns out that our definition is equivalent with the definition given in [4] for a particular infinite dimensional case.

Now, let $L$ be a finite dimensional Lie algebra and $K$ an algebraically closed field of characteristic zero. If $L$ is solvable then there is a chain of ideals in $L$,

$$
0=I_{0} \subset I_{1} \subset \cdots \subset I_{n} \subset L=L,
$$

such that $\operatorname{dim} I_{k}=k(k=1, \cdots, n ; n=\operatorname{dim} L)$. This result is known as the Lie's theorem for abstract Lie algebras [3]. A more sophisticated but equivalent formulation of this result is the following: Every irreducible representation of a solvable Lie algebra on a finite dimensional vector space is necessarily one dimensional [5]. We investigate some infinite dimensional versions of this latter result, valid for operator algebras, as done in [7]. M. S,abac has given in [7] a

Received by the editors December 2, 1971.

AMS (MOS) subject classifications (1970). Primary 17B65, 47D15.

Key words and phrases. Radical, solvable Lie algebras, operator algebras. 
definition for infinite dimensional solvable Lie algebras and has shown that for some special Lie algebras of linear operators, solvable in his sense, when irreducible the dimension of the space is one. We give here a more natural definition and get the definition in [7] as a corollary. At the same time that is a version of Lie's theorem for abstract Lie algebras. We also generalize the result in [7] by using similar techniques.

The author is grateful to S. Halperin for some helpful discussions.

2. The radical of a Lie algebra. Let $L$ be a Lie algebra over a field $K$.

2.1. Definition. An ideal $P$ of $L$ is called primitive if for any ideal $I$ of $L$ with the property $I^{(1)} \subset P$ it follows that $I \subset P$. It is obvious that $L$ itself is a primitive ideal. If $P$ is primitive and $I$ is an ideal of $L$ such that $I^{(n)} \subset P$ for a certain $n \geq 0$ then $I \subset P$; therefore if $P$ is primitive in $L$ then $P$ contains any solvable ideal of $L$. The intersection of any family of primitive ideals is a primitive ideal.

2.2. Lemma. An ideal $P$ of $L$ is primitive if and only if the quotient $L / P$ bas no nontrivial abelian ideal.

Proof. If $P$ is primitive and $I_{1}$ is abelian in $L / P$ then $\phi^{-1}\left(I_{1}\right) \subset P$, where $\phi$ is the canonical mapping of $L$ onto $L / P$, hence $I_{1}=0$. Conversely, if $L / P$ has no nontrivial abelian ideal and $I^{(1)} \subset P$, then $\phi(I)=0$ and $I \subset P$.

2.3. Definition. The intersection $R_{L}$ of all primitive ideals of $L$ is called the radical of $L$.

Let us remark that if $L$ is finite dimensional then $R_{L}$ is the usual radical of $L$. For, it is sufficient to note that the largest solvable ideal of $L$ is primitive.

2.4. Lemma. If the only abelian ideal of $L$ is 0 then $R_{L}=0$.

Proof. Indeed, 0 is in this case a primitive ideal of $L$.

2.5. Proposition. If $P$ is a primitive ideal of $L$ then the radical of the quotient $L / P$ is 0 .

Moreover, $R_{L}$ is the smallest ideal $I$ of $L$ with the property that the radical of $L / I$ is 0 .

Proof. The assertion follow easily from Lemmas 2.2 and 2.4 .

In case when $L$ is finite dimensional we may give a complete description of its primitive ideals.

2.6. Proposition. Let $L$ be a finite dimensional Lie algebra over a field of characteristic zero. An ideal $P$ of $L$ is primitive if and only if $P \supset R_{L}$.

Proof. It is sufficient to show that if $P \supset R_{L}$ then $P$ is primitive. We recall that there is a semisimple subalgebra $S$ of $L$ (Levi subalgebra) such that for every ideal $M$ of $L$ we have 


$$
M=\left(R_{L} \cap M\right)+(S \cap M)
$$

where the sum is direct (for details see [1]). Denote by $p$ the projection of $L$ on $S$. As $R_{L}$ is an ideal then $p$ is a homomorphism. We have $P=R_{L}+S \cap P$ and suppose that $I^{(1)} \subset P$, where $I$ is an ideal in $L$. Then $p I^{(1)}=(p I)^{(1)} \subset S$ and $S$ is semisimple, hence $(p I)^{(1)}=p I$. But $p I^{(1)} \subset S \cap P$, whence $I \subset R_{L}+S \cap P=P$, and $P$ is primitive.

2.7. Proposition. Let $L$ be a finite dimensional Lie algebra over a field of characteristic zero. If $I$ is an ideal of $L$ and $P$ is primitive in $L$ then $P \cap I$ is primitive in $I$.

Proof. Indeed, $P \cap I \supset R_{L} \cap I$ and $R_{L} \cap I$ is the radical of $I$ [1], hence $P \cap I$ is primitive in $I$.

2.8. Definition. We say that $L$ is semisimple when the radical of $L$ is null. We say that $L$ is simple when $L$ is not commutative and the only ideals of $L$ are 0 and $L$. Obviously, when $L$ is simple then $L$ is semisimple.

Let $\left\{H_{\alpha}\right\}_{a \in A}$ be a family of ideals in $L$. Then by $\Sigma_{a \in A} H_{a}$ we mean the ideal generated in $L$ by the family $\left\{H_{\alpha}\right\}_{a \in A}$.

2.9. Proposition. If $L=\Sigma_{a \in A} L_{a}$ and $L_{a}$ are simple ideals of $L$ then $L$ is semisimple.

Moreover, if $I$ is an ideal of $L$ then there is a set of indices $B \subset A$ such that $I=\Sigma_{\beta \in B} L$.

Proof. The proof of this result is quite standard [1] and the second part involves obviously the first one. Let us sketch the proof of the second part. Suppose $L=\Sigma_{a \in A} L$, where we may assume $L_{a} \cap L_{\beta}=0(\alpha \neq \beta)$. If $0 \neq I \subset L$ is an ideal, then for any $\alpha$ we have either $L_{a} \subset I$ or $L_{a} \cap I=0$. Let $B$ be the set of indices $\beta \in A$ such that $L_{\beta} \subset I$. It is easy to see that for any $\alpha \in A$ the following equality holds:

$$
\left\{x \in L ;\left[x, L_{\alpha}\right]=0\right\}=\sum_{\gamma \neq \alpha} L_{\gamma} .
$$

Take an $x \in I, x=x_{a_{1}}+\cdots+x_{a_{n}}$, with $x_{a_{j}} \in L_{a_{j}}(j=1, \cdots, n)$. If $L_{a_{j}} \cap I=0$ then $\left[L_{a_{j}}, I\right]=0$ and $x_{a_{j}}=0$; therefore $I=\Sigma_{\beta \in B} L$.

When $L$ is an $L^{*}$-algebra then $L$ is semisimple in the sense of Definition 2.8 if and only if it is semisimple as defined in [4]. We recall that an $L^{*}$-algebra is a complex Hilbert space which is also a Lie algebra, with the property that for any $x \in L$ there is an $x^{*} \in L$ such that $([x, y], z)=\left(y,\left[x^{*}, z\right]\right)$, for all $y, z \in L$ (where $(x, y)$ is a scalar product of $L$ ). In such an algebra, the Lie product is continuous with respect to the topology of $L$ [4].

For any set $H \subset L$ denote by $\bar{H}$ its closure in the topology of $L$. 
2.10. Proposition. Let $L$ be an $L^{*}$-algebra. Then $L$ is semisimple if and only if $L=\overline{L^{(1)}}$.

Proof. We use some results in [4]. First, every $L^{*}$-algebra is the direct sum of an abelian ideal and $\overline{L^{(1)}}$; therefore if $R_{L}=0$ then $L=\overline{L^{(1)}}$. Conversely, if $L=\overline{L^{(1)}}$ then $L=\Sigma_{a} L_{a}$, where each $L_{a}$ is a noncommutative closed ideal of $L$ which does not contain any proper closed ideal, and the sum is direct in the sense of Hilbert spaces. Noting that an ideal $I$ is commutative if and only if $\bar{I}$ is commutative, we deduce easily that $L$ has no commutative ideal. By Lemma 2.4 we have $R_{L}=0$.

Any $L^{*}$-algebra with the property $L=\overline{L^{(1)}}$ is called in [4] semisimple. Proposition 2.10 shows that our notion is equivalent with the Schue's concept in the class of $L^{*}$-algebras.

3. Quasi-solvable algebras. We are looking for a class of Lie algebras corresponding to the class of solvable algebras in the finite dimensional case, but with some infinite dimensional characteristics.

3.1. Definition. An ideal $I$ of $L$ is quasi-solvable in $L$ if $I=\Sigma_{a} I_{a}$, where $I_{a}$ is a solvable ideal of $L$ for every $a$. More general, the algebra $L$ is quasisolvable if $L$ is quasi-solvable as ideal of $L$.

3.2. Proposition. If $L$ is a quasi-solvable algebra then one bas $R_{L}=L$.

3:3. Proposition. Let $L$ be a quasi-solvable algebra. Then any subalgebra and any bomomorphic image of $L$ is quasi-solvable.

Propositions 3.1 and 3.2 have a very simple proof.

3.4. Proposition. Let $L$ be a quasi-solvable algebra over an algebraically closed field of characteristic zero and I a finite dimensional ideal of $L$. Then there is a chain $\left\{I_{k}\right\}_{k=0}^{n}$ of ideals of $L$ with $\operatorname{dim} I_{k}=k$, where $n=\operatorname{dim} I$, such that

$$
0=I_{0} \subset I_{1} \subset \cdots \subset I_{n}=I
$$

Proof. Let $\phi(x)$ be the restriction of the mapping $D_{x}(y)=[x, y]$ on $I$. Then $x \rightarrow \phi(x)$ is a finite dimensional representation of $L$. If $L_{1}=\phi(L)$ then, by Proposition 3.3, $L_{1}$ is quasi-solvable and finite dimensional, hence solvable. By Lie's theorem, there is an increasing chain $\left\{I_{k}\right\}_{k=0}^{n}$ such that $I_{k}$ are linear subspaces of $I, \operatorname{dim} I_{k}=k$ and $D_{x}\left(I_{k}\right) \subset I_{k}(k=1, \cdots, n)$.

3.5. Definition. Let $L$ be a Lie algebra over an algebraically closed field of characteristic zero. We say that $L$ is $\sigma$-solvable if $L$ is generated by a countable family of finite dimensional solvable ideals.

It is obvious that any $\sigma$-solvable algebra is quasi-solvable. The next result may be considered as a Lie's theorem for abstract Lie algebras. 
3.6. Theorem. Let $L$ be a $\sigma$-solvable Lie algebra. Then there is a sequence $\left\{I_{n}\right\}_{n \geq 0}$ of finite dimensional solvable ideals of $L$ with the properties:

(i) $\operatorname{dim} I_{n}=n(n \geq 0)$;

(ii) $0=I_{0} \subset I_{1} \subset \cdots \subset I_{n} \subset \cdots \subset L$;

(iii) $\bigcup_{n \geq 0} I_{n}=L$.

Proof. We may suppose from the very beginning that $L=\bigcup_{m} H_{m}$ where each $H_{m}$ is a finite dimensional solvable ideal of $L$, and the sequence $\left\{H_{m}\right\}_{m} \geq 1$ is increasing. We suppose also that $H_{m} \neq H_{m+1}$ for every $m>0$. If $\operatorname{dim} H_{1}=1$ then we put $I_{1}=H_{1}$; if not, then we apply Proposition 3.4 to $H_{1}$ and get a chain

$$
0=I_{0} \subset I_{1} \subset \cdots \subset I_{n_{1}}=H_{1},
$$

where each $I_{j}$ is an ideal of $L, \operatorname{dim} I_{j}=j$ and $n_{1}=\operatorname{dim} H_{1}$. Take now $L_{1}=L / H_{1}$ and consider the ideal $\phi_{1}\left(H_{2}\right)$ in $L_{1}$, where $\phi_{1}$ is the canonical mapping of $L$ onto $L_{1}$. If $\operatorname{dim} \phi_{1}\left(H_{2}\right)=1$, we put $I_{n_{1}+1}=H_{2}$; when $\operatorname{dim} \phi_{1}\left(H_{2}\right)=n_{2}>1$, then we apply again Proposition 3.4 to $\phi_{1}\left(H_{2}\right)$ and get an increasing chain $\left\{I_{1, j}\right\}_{j=1}^{n}$ of ideals in $L_{1}$ such that $\operatorname{dim} I_{1, j}=j$ and define $I_{n_{1}+l}=\phi_{1}^{-1}\left(I_{1, l}\right)\left(0<l \leq n_{2}\right)$. This method can be continued and we obtain a sequence $\left\{I_{n}\right\}_{n \geq 0}$ with the desired properties.

The properties (i), (ii), and (iii) are used in [7] in order to define $\infty$-solvable algebras. The above theorem shows that it is possible to have a more concise definition.

4. $\sigma$-solvable algebras of operators. In this section $X$ will be a fixed complex Banach space and $B(X)$ the algebra of all linear continuous operators on $X$.

Let $L$ be a $\sigma$-solvable $L$ ie algebra and $\theta$ a representation of $L$ in $X$, i.e. $\theta$ is a homomorphism of $L$ into $B(X)$. It is easy to see that $\theta(L)$ is also a $\sigma$ solvable Lie algebra, therefore when such features are concerned we may deal only with $\sigma$-solvable subalgebras of $B(X)$.

The proof of the next result has some common ideas with one in [7].

4.1. Theorem. Let $L$ be a $\sigma$-solvable Lie algebra in $B(X)$. Then either $L$ is commutative or $L$ contains a non-null finite dimensional ideal $Q$ such that the elements of $Q$ are commuting quasi-nilpotent operators.

Proof. Suppose that there is no non-null finite dimensional ideal of $L$ whose elements are commuting quasi-nilpotent operators. As $L$ is $\sigma$-solvable, according to Theorem 3.6 there is an increasing sequence of solvable ideals $\left\{I_{n}\right\}_{n} \geq 0$ such that $\operatorname{dim} I_{n}=n$ and $\bigcup_{n} I_{n}=L$. We prove by recurrence with respect to $\bar{n}$ that $\left[I_{n}, L\right]=0$ for any $n \geq 1$. When $n=1$, as $\operatorname{dim} I_{1}=1$ then there is an $a_{1} \in I_{1}$, $a_{1} \neq 0$, such that $\left[x, a_{1}\right]=\lambda_{1} a_{1}$, for any $x \in L$. Suppose $\lambda_{1} \neq 0$ for a certain $x \in L$. Then, according to the Kleinecke-Sirokov theorem [2], the operator $a_{1}=$ $\lambda_{1}^{-1}\left[x, a_{1}\right]$ is quasi-nilpotent, hence $I_{1}$ is a one-dimensional ideal of $L$ having a 
quasi-nilpotent generator and, by our assumption, this is impossible. Suppose now $\left[I_{k}, L\right]=0$ and let us show that $\left[I_{k+1}, L\right]=0$. For, let us choose a basis $a_{1}, \ldots$, $a_{k}, a_{k+1}$ in $I_{k+1}$ such that $a_{1}, \cdots, a_{k} \in I_{k}$. Then for any $x \in L$ we have

$$
\left[a_{k+1}, x\right]=\eta_{1} a_{1}+\cdots+\eta_{k} a_{k}+\eta_{k+1} a_{k+1} \text {. }
$$

As $\left[I_{k}, L\right]=0$, we have $\left[a_{k+1},\left[a_{k+1}, x\right]\right]=0$, hence $q_{x}=\left[a_{k+1}, x\right]$ is quasi-nilpotent, according again to the Kleinecke-Sirokov theorem. If $q_{x}=0$ for any $x \in L$ then $\left[I_{k+1}, L\right]=0$ and this is just what we need. If $q_{x} \neq 0$ for at least one $x \in L$, denote by $Q$ the non-null set of quasi-nilpotent elements of $I_{k+1}$. We have obviously $\left[I_{k+1}, I_{k+1}\right]=0$, hence the elements in $Q$ commute. If $q_{1}, \cdots, q_{m} \in Q$ then $a_{1} q_{1}+\cdots+a_{m} q_{m}$ is again quasi-nilpotent, hence $Q$ is a linear subspace. We have also $[L, Q] \subset\left[L, I_{k+1}\right] \subset Q$, hence $Q$ is an ideal of $L$ and this is impossible. Consequently, $L$ must contain only commuting elements.

4.2. Corollary. Let $L$ be a Lie algebra in $B(X)$ such that $R_{L}$ is $\sigma$-solvable. If the associative algebra with unit generated by $L$ in $B(X)$ is semisimple then $R_{L}$ is equal to the center of $L$.

Proof. Denote by $C_{L}$ the center of $L$. Since $C_{L}$ is commutative we have $C_{L} \subset R_{L}$. On the other hand, as the associative algebra generated by $L$ in $B(X)$ is semisimple, $R_{L}$ may not contain any non-null quasi-nilpotent operator. By Theorem 4.1, $R_{L}$ is commutative. Now, if $x \in L$ and $y \in R_{L}$ then $[x, y] \in R_{L}$ and $[[x, y], y]=0$. By the Kleinecke-Sirokov theorem it follows that $[x, y]$ is quasinilpotent, therefore $[x, y]=0$. Consequently $R_{L}=C_{L}$.

4.3. Corollary. If $L$ is a Lie algebra in $B(X)$ such that the associative algebra with unit generated by $L$ in $B(X)$ is semisimple, then $L$ is $\sigma$-solvable if and only if $L$ is commutative and has a countable algebraic basis.

Proof. If $L$ is commutative and has a countable basis then $L$ is $\sigma$-solvable. Conversely, if $L$ is $\sigma$-solvable then $L$ has a countable basis and $L$ may not contain any quasi-nilpotent operator, hence because of The orem 4.1, it is commutative.

From now on we investigate some infinite dimensional variants of the following statement: Any finite dimensional irreducible representation of a solvable Lie algebra is one dimensional.

Let $L$ be a complex Lie algebra and $\theta$ a representation of $L$ in the Banach space $X$. We say that $\theta$ is irreducible [7] if there is no Banach space $Y$ in $X$, $0 \neq Y \neq X$, such that $\theta(x) Y \subset Y$ for any $x \in L$. A question arises naturally: Is every irreducible representation of a $\sigma$-solvable Lie algebra one dimensional? The answer seems to be related more or less to the classical problem of invariant subspaces. A partial answer was given in [7]. We are going to generalize that result and to point out some other remarks. 
4.4. Definition. A representation $\theta$ of a $\sigma$-solvable Lie algebra $L$ in the Banach space $X$ is called a $d$-representation if

(1) $\theta(x)$ is a decomposable operator [2] on $X$ for any $x \in L$.

(2) If $\theta(x)-\lambda$ is quasi-nilpotent then $\theta(x)-\lambda$ is actually a nilpotent operator, where $\lambda$ is a scalar.

4.5. Theorem. Let $L$ be a $\sigma$-solvable complex Lie algebra and $\theta$ a d-representation of $L$ in the Banacb space $X$. If $\theta$ is irreducible then $\operatorname{dim} X=1$.

Proof. We may suppose $L \subset B(X)$. Since $L$ is $\sigma$-solvatle, because of Theorem 4.1 we have to study two cases:

(a) Suppose $L$ is commutative and denote by $\sigma(x)$ the spectrum of $x$ as operator, where $x \in L$. If there is an $x_{0} \in L$ such that $\sigma\left(x_{0}\right)$ contains at least two points, then there is a maximal spectral space of $x_{0}$ [2] which is different from zero and $X$, say $Y$. Since $L$ is commutative we have $x Y \subset Y$ for any $x \in L$ [2]. Consequently, $Y$ is a proper Banach subspace of $X$ invariant to $L$, and this is impossible. Therefore, for each $x \in L$ there is a complex number $\lambda_{x}$ such that $\sigma(x)=\left\{\lambda_{x}\right\}$, i.e. $x-\lambda_{x}$ is quasi-nilpotent. By our assumption, $x-\lambda_{x}$ is actually nilpotent. If $x \neq \lambda_{x}$ for a certain $x$ then $Y=\left\{\xi \in X ;\left(x-\lambda_{x}\right) \xi=0\right\}$ is a proper Banach subspace of $X$, invariant to $L$. This is again impossible, hence $x=\lambda_{x}$ for any $x \in L$. But in this case any closed subspace of $X$ is invariant to $L$, thus we must have $\operatorname{dim} X=1$.

(b) Suppose that there is a finite dimensional ideal $Q$ of $L$ such that $Q$ contains only commuting quasi-nilpotent operators, hence nilpotent. Let us take a basis $\left\{q_{1}, \cdots, q_{n}\right\}$ in $Q$ and define the space

$$
Y=\bigcap_{k=1}^{n} \operatorname{ker} q_{k} .
$$

Since $q_{k} \neq 0$ for any $k$ we have $Y \neq X$. On the other hand, since $q_{k}(k=1, \cdots$, $n$ ) are commuting nilpotents, $Y \neq 0$. We have also that $Q$ is an ideal of $L$, hence for any $x \in L$ and $q \in Q$ there are some complex numbers $\alpha_{j}^{l}$ such that

$$
q_{l} x=x q_{l}+\sum_{j=1}^{n} \alpha_{j}^{l} q_{j} \quad(l=1, \cdots, n),
$$

therefore if $\xi \in Y$ then $x \xi \in Y$, hence $Y$ is invariant to $L$ and this is not possible. Anyway, we must have $\operatorname{dim} X=1$ and the proof is complete.

4.6. Corollary [7]. Let $L$ be a $\sigma$-solvable complex Lie algebra and $\theta$ a representation of $L$ in the Banacb space $X$, sucb that $\theta(x)$ is a scalar generalized operator [2] for any $x \in L$. If $\theta$ is irreducible then $\operatorname{dim} X=1$.

Proof. It is sufficient to show that $\theta$ is a $d$-representation of $L$ in the Banach space $X$. Indeed, every $\theta(x)$ is scalar generalized hence decomposable [2] and any quasi-nilpotent scalar generalized operator is actually nilpotent [2]. 
4.7. Proposition. Let $L$ be a $\sigma$-solvable complex Lie algebra and $\theta$ a representation of $L$ in $X$ with the properties:

(1) $\theta(x)$ is decomposable for any $x \in X$.

(2) The associative al gebra with unit generated by $\theta(L)$ in $B(X)$ is semisimple.

If $\theta$ is irreducible then $\operatorname{dim} X=1$.

Proof. Assume $L \subset B(X)$. Since the associative algebra generated by $L$ in $B(X)$ is semisimple, according to Corollary 4.3 , we have $L$ commutative. As in the proof of Theorem 4.5, if $\sigma\left(x_{0}\right)$ has at least two points, for a certain $x_{0} \in L$, then there is a proper closed subspace of $X$, invariant to $L$, and this is not possible. If $\sigma(x)=\left\{\lambda_{x}\right\}$ for any $x \in L$ then $x-\lambda_{x}$ is quasi-nilpotent and, by our assumption, we should have $x=\lambda_{x}$. But in this case any closed subspace of $X$ is invariant to $L$, unless $\operatorname{dim} X=1$.

Acknowledgement. The author is grateful for the support of National Research Council Grant of Dr. A. J. Coleman, Head, Department of Mathematics, Queen's University. He would like also to thank the referee for many valuable suggestions which improved the algebraic part of this work.

\section{REFERENCES}

1. N. Bourbaki, Éléments de mathématique. XXVI. Groupes et algèbres de Lie. Chap. 1: Algèbres de Lie, Actualités Sci. Indust., no. 1285, Hermann, Paris, 1960. MR 24 \#A2641.

2. I. Colojoara and C. Foiaș, Theory of generalized spectral operators, Gordon and Breach, New York, 1968.

3. N. Jacobson, Lie algebras, Interscience Tracts in Pure and Appl. Math., no. 10, Interscience, New York, 1962. MR $26 \# 166$.

4. J. R. Schue, Hilbert space methods in the theory of Lie algebras, Trans. Amer. Math. Soc. 95 (1960), 69-80. MR 22 \#8352.

5. Séminaire "Sophus Lie" de l'Ecole Normale Supérieure 1954/55. Théorie des algèbres de Lie. Topologie des groupes de Lie, Secrétariat mathématique, Paris, 1955. MR 17, 384 .

6. I. Stewart, Lie algebras, Lecture Notes in Math., vol. 127, Springer-Verlag, Berlin and New York, 1970. MR 41 \#8483.

7. M. Sabac, Une généralisation du théorème de Lie, Bull Sci. Math. 95 (1971), 53-57.

8. F.'H. Vasilescu, Radical d'une algèbre de Lie, C. R. Acad. Sci. Paris 274 (1972), $536-538$.

INSTITUTE OF MATHEMATICS, ACADEMY OF THE ROMANIAN SOCIALIST REPUBLIC, BUCHAREST, ROMANIA (Current address)

DEPARTMENT OF MATHEMATICS, QUEEN'S UNIVERSITY, KINGSTON, ONTARIO, CANADA 